Plata Durán, M., Pedraza Avella, A., y Ortiz Pimiento, N. (2013). Los factores del capital humano en el estilo de liderazgo de los dirigentes empresariales. Revista Lebret (5). Bucaramanga, Colombia: Universidad Santo Tomás, pp 295-314.

\title{
Los factores del capital humano en el estilo de liderazgo de los dirigentes empresariales del Área Metropolitana de Bucaramanga, Colombia*
}

\author{
Factors of Human Capital in the Leadership Style of Business \\ Managers in the Metropolitan Area of Bucaramanga, Colombia
}

Margarita María Plata Durán', Aura Cecilia Pedraza Avella², Néstor Raúl Ortiz Pimiento

\begin{abstract}
Resumen
El presente estudio tiene como objetivo identificar la relación de los factores de capital humano (experiencia y escolaridad) en el estilo de liderazgo de los gerentes de las empresas del Área Metropolitana de Bucaramanga - AMB (Colombia). Partiendo de la revisión de la literatura, el desarrollo metodológico de la investigación se basa en la aplicación de la teoría de Bass usando un instrumento validado, Cuestionario de Estilos de Liderazgo - CELID, mediante un estudio transversal, de naturaleza descriptiva, exploratoria y relacional a 329 gerentes del Área Metropolitana de Bucaramanga- Colombia. Por otra parte, las características de los gerentes de empresas del Área Metropolitana muestran a un gerente promedio con estilo de liderazgo transformacional, experiencia laboral de más de veinte años y título universitario. Como resultado principal, se evidencian relaciones entre los niveles de escolaridad y la experiencia del gerente con su estilo de liderazgo.
\end{abstract}

\section{Palabras clave}

Gerente, Estilos de Liderazgo, Factores de Capital Humano, Teoría De Bass.

Códigos de Clasificación JEL: M10-J24-M12

\begin{abstract}
This study aims at determining the relationship of the human capital factors (experience and education) in the leadership style of managers of companies in the Metropolitan Area of Bucaramanga - AMB (Colombia). Starting from the review of literature, the methodological development of the research is based on the Bass theory, using the validity survey titled Leadership Styles Questionnaire - CELID, in $a$ transversal, descriptive, exploratory and relational study to 329 managers of AMB-Colombia. On the other hand, the manager characteristics show that the average manager of the Metropolitan Area of Bucaramanga has a transformational leadership style; over twenty years work experience and a university degree. As a main result, there are evident relationships between the educational level and the manager's experience with his/her leadership style
\end{abstract}

\section{Keywords}

Manager, Leadership Styles, Human Capital Factors, Bass Theory.

* $\quad$ Este artículo es resultado del proyecto de investigación "Relación del perfil y estilo de liderazgo el gerente en el éxito de las empresas del Área Metropolitana de Bucaramanga" que se adelanta en el Grupo de Investigación Finance and Management de la Universidad Industrial de Santander, en el que se inscribe el Trabajo de Grado presentado en la Maestría en Ingeniería Industrial por la coautora Margarita Plata Durán.

1 Magister en Ingeniería Industrial de la Universidad Industrial de Santander, Colombia. Docente de Planta en la Facultad de Administración de Empresas de la Universidad Pontificia Bolivariana Bucaramanga, Colombia. Correo electrónico: margarita.plata@upb.edu.co_

2 Doctora en Ciencias Económicas. Docente de planta y Coordinadora de la Maestría en Ingeniería Industrial de la Escuela de Estudios Industriales y Empresariales de la Universidad Industrial de Santander, Colombia. Correo electrónico: acecipe@uis.edu.co

3 Magister en Ingeniería de Sistemas. Docente de planta y Director de la Escuela de Estudios Industriales y Empresariales, de la Universidad Industrial de Santander, Colombia Correo electrónico: nortiz@uis.edu.co 


\section{Introducción}

Las empresas son dirigidas por personas que al interior de las organizaciones transfieren su personalidad, experiencias y capacidades para la obtención de resultados. Por lo tanto, es indispensable estudiar las características de los dirigentes de las organizaciones.

En los consensos de la comunidad científica, la literatura apunta a definir de manera concurrente que el perfil de un gerente es parte fundamental en los resultados organizativos y evidencian la relación del actuar con las habilidades y capacidades que permiten mejorar el ingreso de las empresas (Becker, 1964) ante la presencia de un estilo de liderazgo asociado al perfil del dirigente (Bass, 1990); estas características individuales se han relacionado a factores de capital humano considerando el nivel de estudios, la educación y la experiencia preponderantes en la influencia que los dirigentes infunden a las organizaciones y el direccionamiento de los procesos administrativos (Northouse, 2004). Por ende, se involucra un pensamiento estratégico en donde el planear, organizar, dirigir y controlar forma parte del ejercicio gerencial que comprueba las relaciones entre la personalidad, habilidades y capacidades del gerente, la capacidad de influir en sus subordinados para el cumplimiento de los resultados organizativos y el desempeño de la empresa.

De esta manera, antes de lanzar estrategias de direccionamiento organizacional es necesario identificar estas relaciones y de manera específica conocer el perfil gerencial de quien debe provocar cambios o innovaciones en sus seguidores a partir de la concientización de las metas y resultados organizativos (Bass, 1985), lo cual es una habilidad del líder que logra que sus seguidores antepongan las metas organizativas a las individuales, trasmitiendo de manera efectiva la visión de futuro como parte del cambio al interior de la organización (Bass, 1997); siendo esta capacidad de liderar una característica asociada por diversos autores a la experiencia y el nivel de educación de los dirigentes que ha sido analizada en diferentes contextos.

Es por esto que se plantea la siguiente pregunta de investigación: ¿Cuál es la relación del estilo de liderazgo de los gerentes de las empresas del Área Metropolitana de Bucaramanga - Colombia en los factores del capital humano?

Para responder dicha pregunta, se caracterizaron los gerentes del Área Metropolitana de Bucaramanga (AMB), mediante una investigación de tipo cuantitativo, contando para ello con la participación de una muestra estratificada de 329 dirigentes de empresas.

\section{Factores de capital humano}

El capital humano es el conocimiento que las personas poseen y las capacidades adquiridas (experiencia), se considera un activo para las empresas (Schultz, 1959), 
siendo acuñado en la American Economic Association como sinónimo de educación y formación y abordado en adelante sobre la base de la experiencia laboral y la educación. Estos factores de capital humano han sido estudiados desde la definición de "capital" en la teoría económica, concibiéndose la formación de los individuos como un factor para incrementar la capacidad de producción o elevar el nivel de vida (Smith, 1983). Según la Organización para el Comercio y Desarrollo Económico (OCDE), el capital humano representa "el conocimiento, las competencias y otros atributos que poseen los individuos y que resultan relevantes a la actividad económica" (OCDE, 1999, p.23).

Por otra parte, además de los factores de capital y trabajo, el capital humano es incluido como un tercer factor para explicar fenómenos macroeconómicos como por ejemplo el incremento del ingreso nacional (Becker, 1964; Mincer, 1974). Asimismo, siendo considerada la acumulación de conocimientos y habilidades como causa inmediata del crecimiento económico de las organizaciones. Lewis (1976) y ratificado por Hambrick y Mason (1984), sustentan que las características del líder de la empresa son en parte producto de perfiles relacionados con los factores de capital humano y se consideran como una inversión fundamental para el crecimiento y el bienestar de los países, siendo por esto considerado como un contribuyente en el crecimiento económico, dado que un trabajador con educación tendrá una mayor posibilidad de recibir una mejor remuneración (Becker, 1983). Carpenter, Geletkanycz y Sanders (2004) adicionaron las habilidades y capacidades del líder a las variables tradicionales de capital humano como son la educación y experiencia.

EI Liderazgo. El líder está relacionado con los términos poder y autoridad como la capacidad que tiene una persona o agente para influenciar a otra (Mintzberg, 1993). Entre los principales enfoques teóricos que han sido aplicados para medir el liderazgo, se encuentran el enfoque de los rasgos, el enfoque conductual, el enfoque situacional, el enfoque de Bass, entre otros.

El enfoque de los rasgos está enmarcado en las "teorías del gran hombre" (Stogdill, 1948) y se basa en establecer características estables de personalidad que diferencian a los líderes de quienes no lo son, basados en que los hombres nacen con dicha cualidad (Stogdill, 1948; Mann, 1959; Lord, De Vader y Alliger, 1986; Kirkpatrick y Locke, 1991). Sin embargo, a nivel organizacional se orienta con temáticas como las competencias laborales (Masten y Coastworth, 1998; Sternberg, 1998) en donde estas competencias han sido adquiridas con el tiempo y relacionadas con los factores de capital humano (experiencia y educación).

El enfoque conductual nace como resultado de estudios realizados en la Ohio State University y centra sus investigaciones en determinar las conductas de los líderes y su relación con el liderazgo (Hemphill y Coons, 1957; Cartwright y Zander, 1960; Stogdill, 1963) mediante la identificación de conductas individuales y no patrones de comportamiento. 
El enfoque situacional basa sus explicaciones en la teoría del Camino - Meta (Evans, 1970; House, 1971; House y Dessler, 1974; House y Mitchell, 1974) y en la teoría de la contingencia (Fiedler, 1967); este enfoque asegura que el líder efectivo adapta su estilo de liderazgo acorde al contexto en el que se desenvuelve (Hersey, Blanchard y Johnson, 1969), se considera un enfoque teórico y con debilidades en su medición, sin embargo es considerado como un inicio de las teorías no clásicas del liderazgo.

La teoría o enfoque de Bass (1985) es uno de los más estudiados y adaptado a diferentes contextos, parte de la teoría del líder carismático y transformacional (House, 1977; Burns, 1978). Su precursor fundamentó su enfoque en la identificación de dos estilos de liderazgo: el líder transformacional y el líder transaccional, siendo definido el liderazgo transformacional como un proceso en el cual los líderes influyen sobre sus seguidores mediante la motivación en la obtención de resultados organizativos. Por otra parte, el líder influencia a sus seguidores mediante la motivación de los intereses personales de estos, el líder tiene un tipo de liderazgo transaccional. Finalmente la teoría incluyó un estilo de liderazgo ineficaz denominándolo laissez faire que significa la ausencia del liderazgo.

Actualmente, uno de los modelos base de una gran cantidad de investigaciones en la comunidad científica es el modelo de liderazgo de Bass (1985), dada la gran aplicabilidad que tiene en el ámbito organizacional con la aplicación de un instrumento estándar validado, el Multifactor Leadership Questionnaire MLQ (Bass, 1997). Este cuestionario ha sido adaptado generando versiones en español como lo es el Cuestionario de Estilos de Liderazgo - CELID. Esta categorización fue aplicada para conocer el tipo de liderazgo que se distinguía en 72 pelotones del Ejército de los Estados Unidos (Bass, Avolio, Jung, y Berson, 2003) y los resultados evidenciaron la interacción entre el líder y el resto de los miembros de organización en dos categorías, el liderazgo transaccional y el liderazgo transformacional.

En el contexto hispano se han realizado estudios de liderazgo dentro de los cuales se encuentran Molero, Cuadrado, Navas y Morales (2007), Moriano, Molero y Mangin (2011), Castro y Nader (2004), Castro y Casullo (2005), Nader y Castro (2010), Pedraja, Rodríguez y Rodríguez (2006) y Ogliastri (2005) aplicando las teorías existentes y asociando el estilo de liderazgo con características de perfil como son las de capital humano como se muestra a continuación. Cabe señalar que el modelo Bass (1997) es el utilizado en la mayor cantidad de investigaciones sobre liderazgo en Hispanoamérica que se presentan en las bases científicas de calidad tales como ISI Knowledge, Scopus, Jstor.

EI liderazgo y los factores de capital humano. Dentro de la literatura científica, existen diversos estudios que han abordado el interés por interpretar el efecto del perfil del gerente y de manera particular, la relación existente entre los factores de capital humano y el estilo de liderazgo. 
En Chicago (Estados Unidos) se realizó un estudio a 832 empresarias cuyos negocios crecieron rápidamente, encontrando entre algunos rasgos distintivos y correlacionados de manera significativa el estilo de liderazgo, las características de la empresa (tamaño, sector) y del empresario como la experiencia (Gundry y Welsch, 2001)

En Grecia (Spanos y Lioukas, 2001) se estudió el perfil del líder en relación con el impacto relativo de la industria y los factores específicos de la empresa. En los resultados se encuentra una alta relación entre las fuerzas de la industria, que actúan como causa directa en el rendimiento del mercado, y la rentabilidad de los activos empresariales, al igual que una alta relación entre el liderazgo del gerente y el perfil del líder (características de su personalidad, capacidades y habilidades por experiencias previas y nivel de educación).

En Israel, se realizó una de las investigaciones más completas de los factores que inciden en el éxito empresarial mediante un trabajo de campo con 93 ejecutivos de empresas (escogidos por conveniencia), en éste se investigó la interrelación de variables organizativas de rendimiento, con variables de capacidades de gestión entre las cuales se encontraba el liderazgo, factores externos de percepción de incertidumbre y las capacidades de los ejecutivos (entre las cuales se encontraba el nivel de escolaridad y la experiencia). Los resultados mostraron que las capacidades de gestión de quienes dirigen a las organizaciones afectan en gran medida resultados de la empresa y estas a su vez, se encontraban relacionadas con las capacidades de los dirigentes (Carmeli y Tishler, 2006).

En Alemania, Rauch y Frese (2007), analizaron mediante un modelo los efectos de los rasgos de personalidad en la puesta en marcha de una empresa, encontrando en los resultados la existencia de rasgos coincidentes con el espíritu empresarial y una relación significativa entre la personalidad, factores de capital humano (experiencia previa del emprendedor, educación y tipo de liderazgo).

En la India, utilizando una muestra de 195 empresas pequeñas y medianas empresas de la industria de outsourcing, se analizó la flexibilidad estratégica de las relaciones entre la personalidad (liderazgo y características de capital humano) y el desempeño de la empresa, encontrando resultados que no sólo ponen de relieve la importancia de las características personales del Director General en la conducción de la flexibilidad estratégica, sino que también indica cómo cada faceta de la personalidad de director general potencia o inhibe la flexibilidad de la estrategia (Nadkami y Hermann, 2010).

Adicionalmente, en una investigación aplicada a los líderes de 25 unidades estratégicas del hospital de psiquiatría de Kaunas en Lituania (Kaminskas, Bartkus y Pilinkus, 2011), se analizó la interacción entre un líder y los seguidores, comparando las características de los líderes (características socio- demográficas y de capital 
humano). En los resultados, se evidenciaron perfiles característicos en los líderes que motivaban la creación de confianza y respeto mutuo con todos los subordinados.

En China (Wang, Tsui y Xin, 2011) se exploraron 125 empresas para establecer la relación entre los comportamientos de liderazgo (la creatividad y la toma de riesgos, la relación y comunicación, benevolencia, articulación de una visión, autoritarismo, vigilancia y control), las actitudes del CEO (apoyo organizacional percibido, compromiso organización, imparcialidad en resultados e imparcialidad en procesos), el desempeño de la empresa desde su rentabilidad y las habilidades y capacidades de los líderes (tiempo laborado en la empresa, tiempo de vida laboral, estudios realizados y edad), encontrando entre los comportamientos de liderazgo la vinculación directa con las habilidades y capacidades, así como con los resultados empresariales.

En Chipre, Eyupoglu y Saner (2011) caracterizaron las mujeres empresarias del norte de Chipre con una muestra de 176 mujeres empresarias exitosas; la investigación encontró, mediante la estadística descriptiva, que los factores que influían en el éxito empresarial eran factores del perfil personal (estilo de liderazgo, nivel educativo, experiencia en el sector y experiencia laboral anterior) y factores propios de las características de su negocio (sector económico y tamaño de la empresa).

En el año 2012 en Taiwán, se investigaron las relaciones de estilo de liderazgo del gerente, el perfil y su impacto en el desempeño de los proyectos al interior de las organizaciones. Los análisis sugieren que el liderazgo transformacional puede ser positivamente relacionado con la comunicación del equipo y la colaboración, y a su vez con el perfil de líder del proyecto (Yang, Wu, Wang, y Chin, 2012).

Dentro de las investigaciones empíricas más relevantes que se han basado en el modelo de Bass en el contexto hispano, está un estudio donde se analizó a 147 dirigentes de empresas españolas (Molero, Cuadrado, Navas y Morales, 2007) evidenciando que el liderazgo transformacional tenía una alta relación positiva y significativa con los resultados de la organización y se relacionada directamente con mayores experiencias previas y nivel de educación de los sujetos. La validez del instrumento (Moriano, Molero y Mangin, 2011) se realizó través de un análisis factorial confirmatorio con un grupo de 600 directores de empresas mostrando un cuestionario con una fiabilidad y validez concurrente con un coeficiente de confiabilidad en las variables superior a 0,83. En América Latina el modelo Bass se ha adaptado al contexto (Castro y Nader, 2004) como el Cuestionario de Estilos de Liderazgo (CELID), el cual se ha validado mediante estudios en diferentes países como los realizados en Argentina (Castro y Casullo, 2005; Nader y Castro, 2010) quienes evidenciaron la influencia existente de los valores en el estilo de liderazgo tanto transformacional como transaccional con los aspectos de personalidad y de capital humano. En Chile, Pedraja, Rodríguez y Rodríguez (2006), evaluaron la influencia del liderazgo en las empresas encontrando que el estilo de liderazgo transformacional se encontraba correlacionado con la experiencia laboral y escolaridad. 
En Colombia, el acceso a las investigaciones realizadas sobre el tema y a sus resultados es limitado y los artículos productos de éstas no son divulgados en bases de datos reconocidas o revistas de alto impacto. Dentro de la literatura gris, se estudiaron las características de liderazgo organizacional en Colombia (Ogliastri, 2005) para el estudio Globe, investigación realizada en sesenta y cuatro países y 32 lenguas distintas. En ella se entrevistaron 72 empresas con desempeño a nivel financiero sobresaliente en la ciudad de Bogotá pertenecientes a tres sectores empresariales y se identificaron las diferencias entre el líder, el gerente y tres elementos del liderazgo (relaciones humanas y personal, visión, estilo gerencial) y tres aspectos complementarios (integridad, actuación ante una crisis y fijación de objetivos). En el Área Metropolitana de Bucaramanga no se evidencian estudios similares a los realizados en otros países donde se relaciona el liderazgo de los gerentes con los factores de capital humano de sus organizaciones.

\section{Metodología}

Esta Investigación se desarrolló de manera transversal mediante un estudio no experimental cuantitativo, explorando la relación entre el desempeño empresarial y las características de capital humano. El análisis de los datos se realizó con el programa estadístico SPSS versión 18, que permitió caracterizar a los gerentes del Área Metropolitana de Bucaramanga - Colombia mediante la Estadística Descriptiva. La población objetivo son los gerentes de las empresas del Área Metropolitana de Bucaramanga. Las variables de estudio son el estilo de liderazgo, y las variables correspondientes al capital humano son la experiencia y el nivel de escolaridad A partir de un muestreo estratificado por sectores económicos se entrevistaron 329 gerentes. Para ello, se utilizó la prueba existente y válida CELID -A, que identifica los perfiles de liderazgo transformacional, transaccional, y Laissez Faire, indagando de manera adicional sobre los factores de capital humano de cada dirigente (nivel de escolaridad, experiencia laboral, experiencia en el sector y experiencia en la empresa).

El Cuestionario de Estilos de Liderazgo - CELID A (Castro y Nader, 2004) consta de 34 ítems que operacionaliza la teoría de Bass (Bass, 1997). El instrumento es una adaptación española del MLQ (Multifactorial Leadership Questionnaire) realizada en Argentina (Castro y Nader, 2004). La versión utilizada presenta fiabilidad y validez adecuada medida mediante el coeficiente Alpha de Cronbach, el cual se encuentra entre 0.73 y $0.72^{4}$. Las respuestas se encuentran en una escala de 1 a 5 en cada una de las 34 afirmaciones, siendo 1 en total desacuerdo y 5 en total acuerdo;

4 El coeficiente Alfa de Cronbach, verifica la consistencia del instrumento desde las dimensiones de los estilos de Liderazgo Transformacional y Transaccional, por tanto el estilo Liderazgo Laissez Faire, dada su unidimensional, no la requiere. 
una vez aplicada la prueba, esta permite obtener una puntuación promedio para cada uno de los estilos de liderazgo. Este resultado obtenido es transformado a percentiles y comparado con el baremo o escala estándar para identificar el estilo de liderazgo del gerente.

\section{Análisis de datos}

La caracterización de las empresas teniendo en cuenta el sector al cual pertenecen las empresas, observa una participación representativa del sector comercio (49\%), seguido por los sectores de empresas de servicios, industriales y primario con un $26 \%, 22 \%$ y $3 \%$ respectivamente; estos resultados muestran la coherencia con la realidad empresarial regional (ver Tabla 1), con lo cual se refleja la representatividad de muestra y por ende los resultados.

Tabla 1. Composición sectorial de las empresas del AMB

\begin{tabular}{lccc}
\hline \multicolumn{1}{c}{ Sector } & Frecuencia & Porcentaje & $\begin{array}{c}\text { Porcentaje } \\
\text { Acumulado }\end{array}$ \\
\hline Primario & 10 & $3 \%$ & $3 \%$ \\
\hline Industrial & 71 & $22 \%$ & $25 \%$ \\
\hline Comercio & 162 & $49 \%$ & $74 \%$ \\
\hline Servicios & 86 & $26 \%$ & $100 \%$ \\
\hline Total & $\mathbf{3 2 9}$ & $\mathbf{1 0 0} \%$ & \\
\hline
\end{tabular}

Fuente: Autores.

En la Tabla 2 se observa que las empresas de tamaño pequeño tienen una alta participación en el Área Metropolitana de Bucaramanga.

Tabla 2. Composición de las empresas del AMB por tamaño

\begin{tabular}{lccc}
\hline \multicolumn{1}{c}{ Tamaño } & Frecuencia & Porcentaje & $\begin{array}{c}\text { Porcentaje } \\
\text { Acumulado }\end{array}$ \\
\hline Microempresa & 144 & $44 \%$ & $44 \%$ \\
\hline Pequeña & 136 & $41 \%$ & $85 \%$ \\
\hline Mediana & 37 & $11 \%$ & $96 \%$ \\
\hline Grande & 12 & $4 \%$ & $100 \%$ \\
\hline Total & $\mathbf{3 2 9}$ & $\mathbf{1 0 0 \%}$ & \\
\hline
\end{tabular}

Fuente: Autores. 


\section{Los factores de capital humano en las empresas del Área Metropolitana de Bucaramanga - AMB Colombia}

Caracterización de la educación de los gerentes del AMB. En el nivel de escolaridad del gerente del Área Metropolitana de Bucaramanga tiene una mayor representación el título universitario (42\%), seguido del nivel de postgrado (22\%), técnico o tecnológico $(17 \%)$, bachiller $(13 \%) \mathrm{y}$, con una menor representación los que no poseen título de bachiller (6\%), como se observa en la Figura 1.

Figura 1. Nivel de escolaridad de los gerentes del $A M B$

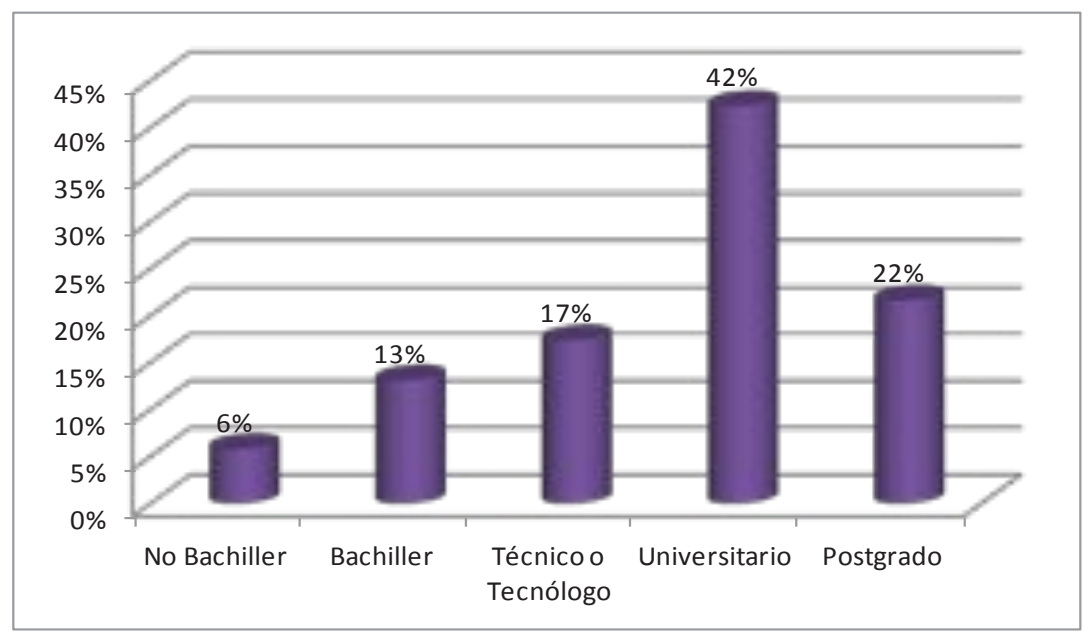

Fuente: Autores.

Adicionalmente, se encuentra que los gerentes del Área Metropolitana que poseen un nivel de escolaridad universitario, han obtenido mayoritariamente el título de Administradores de Empresas (37,1\%), seguido por las carreras de Ingeniería Industrial (8,6\%), Ingeniería civil (7,9\%), Contaduría Pública (5,7\%), Derecho (5,7\%). Las demás carreras universitarias tienen representaciones bajas (ver Figura 2). 
Figura 2. Título universitario de los gerentes del $A M B$

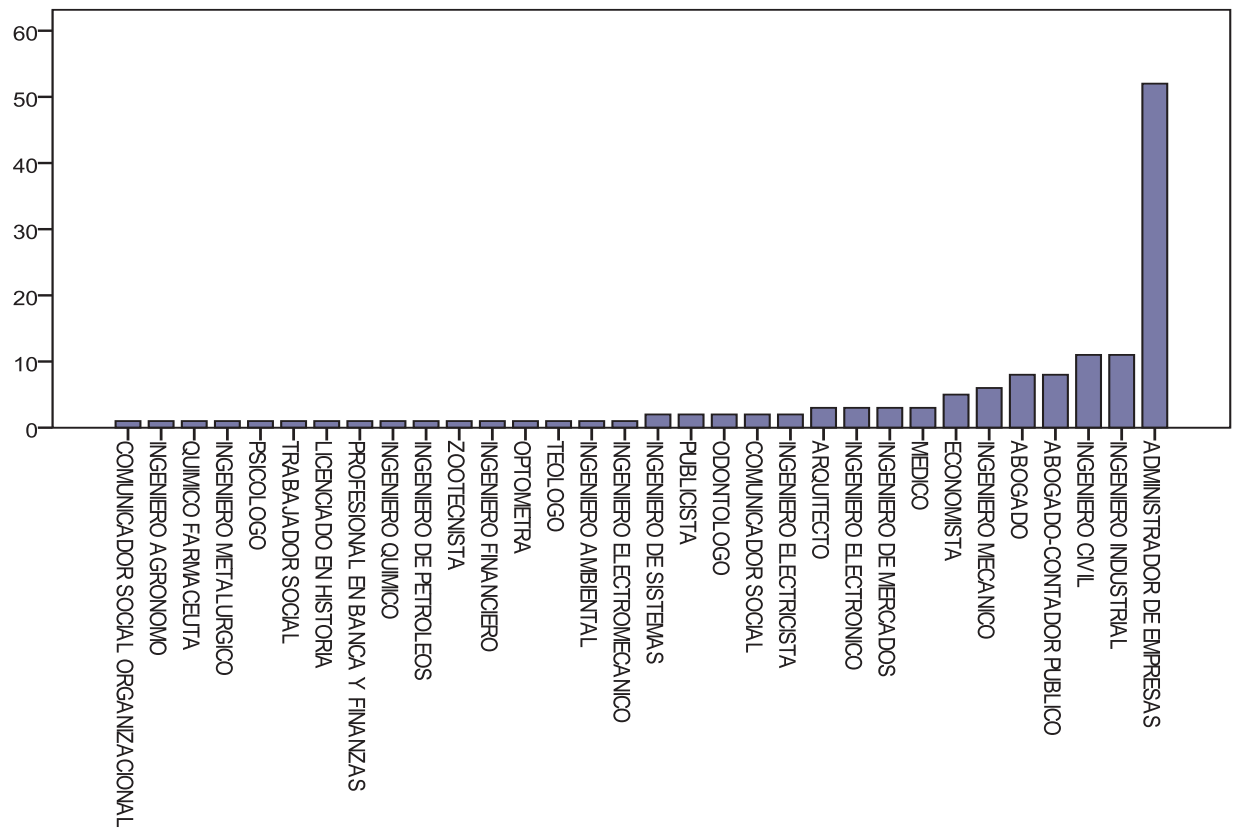

Fuente: Autores.

Los gerentes del Área Metropolitana que poseen un nivel de escolaridad de postgrado, han obtenido en su mayoría el título de MBA (18\%), con menor participación de quienes han realizado especializaciones en finanzas (7\%), alta gerencia $(5 \%)$, gerencia $(5 \%)$, recursos humanos $(4 \%)$, gerencia financiera $(4 \%) y$ mercadeo (4\%). Los demás postgrados presentan representaciones bajas.

Existe una relación directa entre los años de vida laboral y la posibilidad de desempeñarse como gerente en el AMB. Una mayoria de gerentes tiene más de 20 años de experiencia y los porcentajes se estreucturan de forma descendente en los grupos que tienen entre los 15 y 20 años, entre los 10 y 15 años y asi sucesivamente, (ver Figuras 3 y 4 ) 
Figura 3. Experiencia laboral de los gerentes del $A M B$

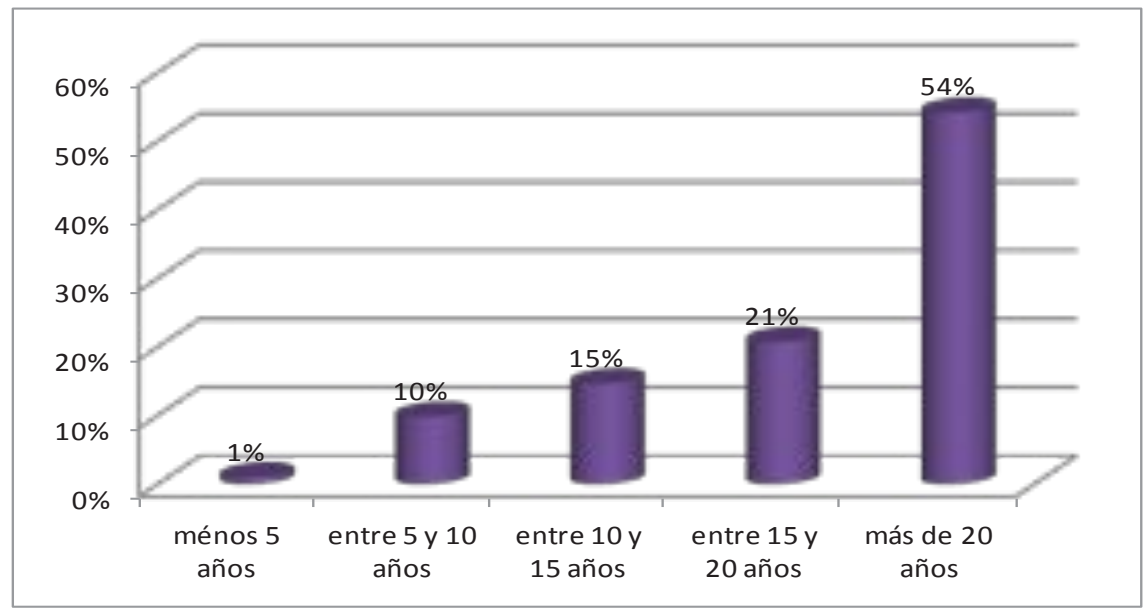

Fuente: Autores.

Figura 4. Experiencia en el sector de los gerentes del AMB

C Más de 20 años,

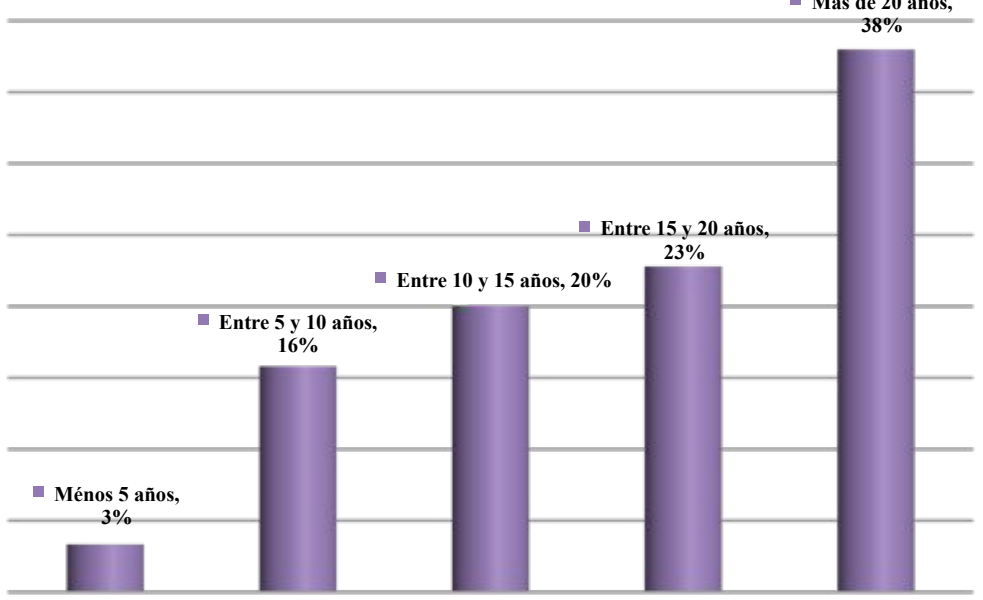

Fuente: Autores. 
Las diferencias existentes en la experiencia laboral de los dirigentes en la empresa donde trabaja actualmente se muestran en la Figura 5, encontrándose que mayoritariamente tienen una experiencia entre 5, 10 años y 20 años, seguido por el rango de 10 y 15 años y el rango entre 15 y 20 años en menor participación quienes poseen experiencia menor a 5 años.

Figura 5. Experiencia en la empresa de los gerentes del AMB

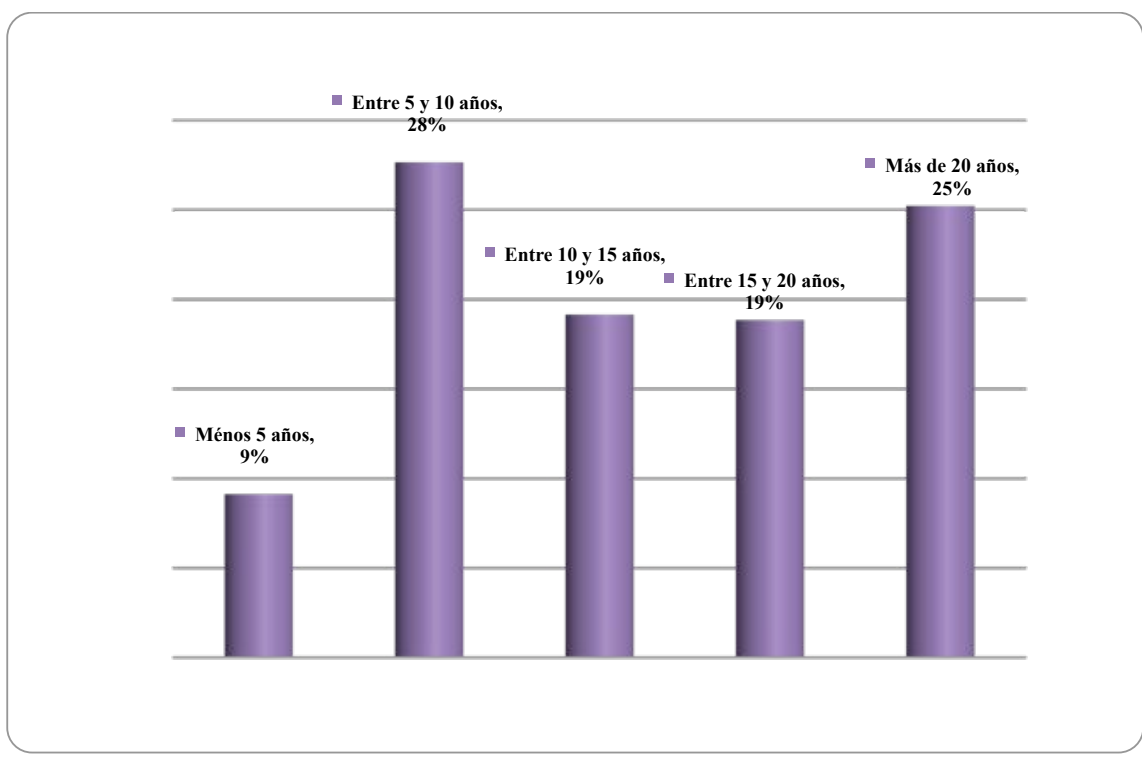

Fuente: Autores.

Estilo de Liderazgo. Como primer análisis, mediante el cálculo de las frecuencias ${ }^{5}$, se observó el estilo de liderazgo con mayor representación, identificándose que los gerentes del Área Metropolitana de Bucaramanga están representados por líderes transformacionales (Ver Figura 6).

$5 \quad$ El instrumento permite medir el estilo de liderazgo y su nivel (alto, medio y bajo) que corresponde al nivel de intensidad del estilo de liderazgo. Los resultados aquí presentados son aquellos en los cuales el estilo de liderazgo presenta un nivel alto o predominante. 
Figura 6. Frecuencias relativas del estilo de liderazgo de los gerentes del AMB

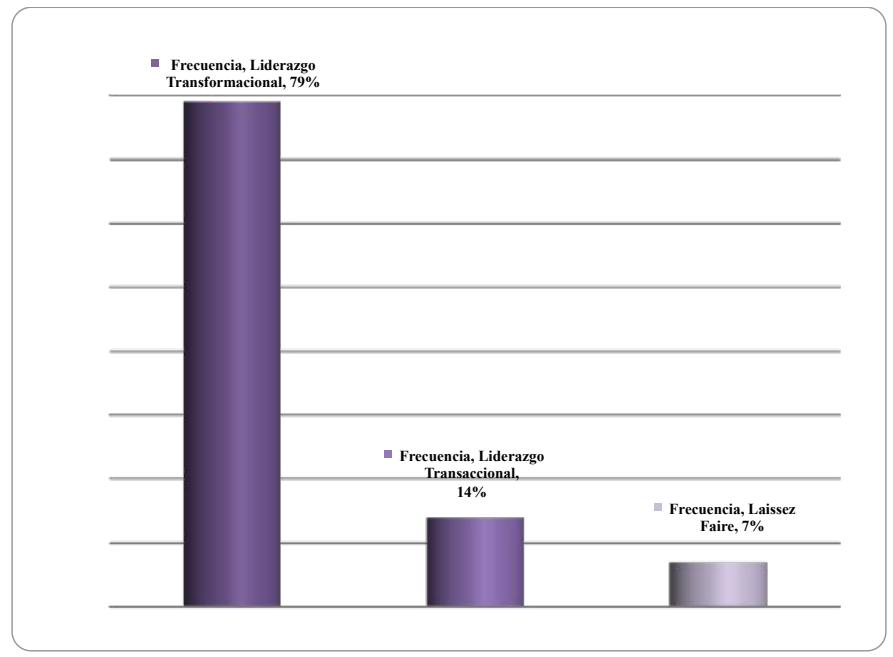

Fuente: Autores.

Con el fin de ratificar la tendencia del estilo de liderazgo se realizó una comparación por medio del estadístico t, se encuentra que existe una diferencia significativa entre todos los estilos de liderazgo con un $\mathrm{p}<0,01$. Lo anterior corrobora los resultados expuestos en las Tablas anteriores en donde el estilo predominante es el transformacional y el menos utilizado es el laissez faire (ver Tabla 3).

Tabla 3. Prueba t entre estilos de liderazgo de gerentes del AMB

\begin{tabular}{lc}
\hline \multicolumn{1}{c}{ Estilos de Liderazgo } & Valor t-student \\
\hline Trans formacional-Transaccional & $29,296^{* * *}$ \\
\hline Trans formacional-Laissez faire & $68,025^{* * *}$ \\
\hline Transaccional-Laissez faire & $23,731^{* * *}$ \\
\hline
\end{tabular}

****Significativo al $\mathrm{p}<0,01$

Fuente: Autores.

Relación del capital humano con el estilo de liderazgo. Teniendo en cuenta la relación del estilo de liderazgo con la escolaridad de los gerentes del Área Metropolitana de Bucaramanga, se identifica que los gerentes con un grado de escolaridad universitario son más lideres transformacionales, mientras quienes no son bachilleres o poseen grado de bachiller presentan ausencia de liderazgo. Por otra 
parte, quienes son técnicos o tecnólogos y quienes tienen un nivel de escolaridad de postgrado son mayoritariamente líderes transaccionales (ver Tabla 4).

\section{Tabla 4. Relación del estilo de liderazgo con la escolaridad} de los gerentes de empresas del AMB

\begin{tabular}{|c|c|c|c|c|c|c|c|}
\hline & & & & & & & \\
\hline & & \multicolumn{4}{|c|}{ Escolaridad } & \multirow[b]{2}{*}{ Postgrado } & \multirow[b]{2}{*}{ Total } \\
\hline & & No Bachiller & Bachiller & $\begin{array}{l}\text { Técnico o } \\
\text { Tecnólogo }\end{array}$ & Universitario & & \\
\hline \multirow{4}{*}{$\begin{array}{l}\text { Estilo de } \\
\text { Liderazgo }\end{array}$} & Liderazgo Transformacional & $1 \%$ & $2 \%$ & $13 \%$ & $46 \%$ & $18 \%$ & 210 \\
\hline & Liderazgo Transaccional & $11 \%$ & $43 \%$ & $39 \%$ & $30 \%$ & $43 \%$ & 77 \\
\hline & Laissez Faire & $50 \%$ & $82 \%$ & $18 \%$ & $18 \%$ & $23 \%$ & 42 \\
\hline & Total & $6 \%$ & $13 \%$ & $17 \%$ & $42 \%$ & $22 \%$ & 329 \\
\hline
\end{tabular}

Fuente: Autores

Para analizar la existencia de relación entre las variables, escolaridad y estilo de liderazgo, se utilizó el estadístico chi-cuadrado en donde se rechaza la hipótesis de independencia al tener un nivel de significancia inferior a 0,05 y se considera que las variables estilo de liderazgo y nivel de escolaridad están relacionadas (ver Tabla 5).

Tabla 5. Estadístico Chi-Cuadrado Estilo de Liderazgo - Escolaridad

Test Pearson Chi- Cuadrado

\begin{tabular}{lrrr}
\cline { 2 - 4 } & Valor & df & Nivel de Significancia \\
\hline Escolaridad & 25,878 & 8 &, 001 \\
\hline
\end{tabular}

Fuente: Autores

Teniendo en cuenta la experiencia laboral, se observa que los gerentes con experiencia laboral menor a 10 años, son en mayor medida líderes transaccionales y cuando su experiencia se encuentra en 10 y 20 años son más líderes transformacionales; sin embargo, al tener más de 20 años de experiencia los dirigentes pasan a no ser líderes (ver Tabla 6) .

Tabla 6. Relación del estilo de liderazgo con la experiencia laboral de los gerentes de empresas del $A M B$

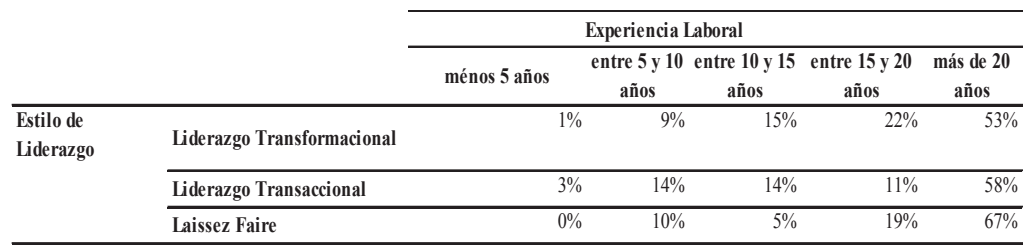

Fuente: Autores 
De manera particular con respecto a la experiencia en el sector y la empresa se observan comportamientos similares, en donde los gerentes con experiencia menor a 5 años y mayor a 20 años son representativamente no líderes, mientras que quienes tienen experiencia entre 5 y 10 años junto con el rango entre 15 y 20 años son principalmente líderes transaccionales; con respecto al rango de 10 y 15 años se observan gerentes caracterizados por un liderazgo transformacional (ver Tablas 7 y 8 ).

Tabla 7. Relación del estilo de liderazgo con la experiencia en el sector de los gerentes de empresas del $A M B$

\begin{tabular}{|c|c|c|c|c|c|c|}
\hline & & \multirow{2}{*}{\multicolumn{4}{|c|}{ Experiencia en el Sector }} & \multirow[b]{3}{*}{$\begin{array}{c}\text { más de } 20 \\
\text { años }\end{array}$} \\
\hline & & & & & & \\
\hline & & ménos 5 años & $\begin{array}{c}\text { entre } 5 \text { y } 10 \\
\text { años }\end{array}$ & $\begin{array}{c}\text { entre } 10 \text { y } 15 \\
\text { años }\end{array}$ & $\begin{array}{c}\text { entre } 15 \text { y } 20 \\
\text { años }\end{array}$ & \\
\hline \multirow{3}{*}{$\begin{array}{l}\text { Estilo de } \\
\text { Liderazgo }\end{array}$} & Liderazgo Transformacional & $3 \%$ & $15 \%$ & $20 \%$ & $21 \%$ & $36 \%$ \\
\hline & Liderazgo Transaccional & $3 \%$ & $28 \%$ & $19 \%$ & $31 \%$ & $47 \%$ \\
\hline & Laissez Faire & $5 \%$ & $5 \%$ & $19 \%$ & $29 \%$ & $48 \%$ \\
\hline
\end{tabular}

Fuente: Elaboración propia de los autores

Tabla 8. Relación del estilo de liderazgo con la experiencia en la empresa de los gerentes de empresas del $A M B$

\begin{tabular}{|c|c|c|c|c|c|c|}
\hline & & & & & & \\
\hline & & \multicolumn{4}{|c|}{ Experiencia en la Empresa } & \multirow[b]{2}{*}{$\begin{array}{l}\text { más de } 20 \\
\text { años }\end{array}$} \\
\hline & & ménos 5 años & $\begin{array}{c}\text { entre } 5 \text { y } 10 \\
\text { años }\end{array}$ & $\begin{array}{c}\text { entre } 10 \text { y } 15 \\
\text { años }\end{array}$ & $\begin{array}{c}\text { entre } 15 \text { y } 20 \\
\text { años }\end{array}$ & \\
\hline \multirow{3}{*}{$\begin{array}{l}\text { Estilo de } \\
\text { Liderazgo }\end{array}$} & Liderazgo Transformacional & $8 \%$ & $26 \%$ & $21 \%$ & $17 \%$ & $24 \%$ \\
\hline & Liderazgo Transaccional & $11 \%$ & $44 \%$ & $17 \%$ & $31 \%$ & $25 \%$ \\
\hline & Laissez Faire & $14 \%$ & $19 \%$ & $5 \%$ & $29 \%$ & $38 \%$ \\
\hline
\end{tabular}

Fuente: Autores

Igualmente, al analizar la relación existente entre el liderazgo y la experiencia se observa que las variables se encuentran relacionadas y por tanto ejercen influencia (ver Tabla 9)

Tabla 9. Estadístico Chi-Cuadrado Estilo de Liderazgo - Experiencia

\begin{tabular}{lcccr} 
& \multicolumn{4}{c}{ Test Pearson Chi- Cuadrado } \\
\cline { 2 - 5 } & Valor & df & Nivel de Significancia \\
\hline Experiencia Laboral & 31,613 & 8 &, 024 \\
\hline Experiencia en el Sector & 43,980 & 8 &, 020 \\
\hline Experiencia en la Empresa & 96,560 & 8 &, 029 \\
\hline
\end{tabular}

Fuente: Autores 


\section{Conclusiones}

Este estudio ofrece una estimación del perfil de los gerentes del Área Metropolitana de Bucaramanga-Colombia, haciendo énfasis en los factores de capital humano (experiencia y nivel de escolaridad) y su relación con el estilo de liderazgo.

Con respecto al estilo de liderazgo en los gerentes del Área Metropolitana de Bucaramanga, se observa que el predominante es el transformacional y el de menor preferencia el estilo laissez faire; estos datos son coincidentes con las investigaciones realizadas por Molero et al. (2007) y Nader et al. (2010). Esto significa que a nivel general los gerentes del Área Metropolitana de Bucaramanga muestran preferencia manifiesta por uno de los tres estilos de liderazgo evaluados por la prueba CELID - A, datos que son coincidentes con las investigaciones realizadas por Molero et al. (2007), Castro y Nader (2004) y Nader et al. (2010).

Acorde a los objetivos propuestos, se evidencia que existe a nivel regional un perfil característico del dirigente lo cual se direcciona hacia el planteamiento de Hambrick y Mason (1984). Se identifica que el gerente del Área Metropolitana de Bucaramanga posee título universitario y tiene experiencia laboral y en el sector superior a 20 años, y en la empresa en el rango entre 5 y 10 años.

Al relacionar la escolaridad con el estilo de liderazgo, se encuentra que la preparación académica superior (técnico, tecnólogo, universitario o postgrado) conlleva al gerente a poseer algún estilo de liderazgo (transformacional o transaccional), contrario a quienes son bachilleres o no quienes estarían representados por la ausencia de transacción de cualquier clase, lo cual caracteriza al laissez faire en donde se evita tomar decisiones, no tiene responsabilidad y no usa su autoridad; similares resultados obtenidos por Castro y Nader (2004). Asimismo, los gerentes cuyo nivel de escolaridad es universitario están caracterizados por un liderazgo transformacional y por tanto procuran niveles de conciencia en los seguidores acerca del valor y la importancia de lograr los resultados asignados y la manera de alcanzarlos, estimulan a sus seguidores a tener ideas innovadoras y creativas e inspiran espíritu de grupo. Los líderes cumplen una función orientadora. Por otra parte, los gerentes con un nivel de escolaridad técnico o tecnólogo y postgrado son mayoritariamente líderes transaccionales y se caracterizan por el intercambio de premios y castigos, y comprenden a todos los estilos clásicos de liderazgo, interviniendo sólo cuando hay que hacer correcciones o cambios en las conductas de los seguidores.

Relacionando la experiencia con el estilo de liderazgo, se observa una clara tendencia del estilo de liderazgo del gerente acorde a los años laborales en el sector o en la empresa, siendo concurrente que cuando el dirigente tiene entre 10 y 20 de experiencia laboral y entre 10 y 15 años de experiencia en el sector, en la empresa es principalmente líder transaccional, mientras que cuando su experiencia (laboral, sector y empresa) supera los 20 años y experiencia en el sector y en la empresa menor a 5 años, se caracteriza por poseer un estilo representado por la ausencia de 
transacción de cualquier clase o laissez faire. Por otra parte, cuando los gerentes tienen experiencia laboral entre 5 y 10 años, experiencia en el sector y en la empresa en los rangos de 5 a 10 años y 15 a 20 años en su mayoría son líderes transaccionales; estos resultados son coincidentes con los obtenidos por Carpenter et al. (2004) en donde las habilidades y capacidades del líder se relacionan con el nivel educativo de éste.

Además, la existencia de diferencias culturales entre regiones, señaladas por diversos autores como Schwartz (2001), Gundry y Welsch (2001), Javidan y House (2002) y Yang et al. (2012), hace necesario dar inicio a este tipo de estudios en Colombia en otros contextos mediante un desarrollo científico, lo cual permitirá la posibilidad de comparar los resultados.

Para dar continuidad a esta investigación, es importante realizar este estudio desde otros contextos (niveles jerárquicos dentro de la organización o en un subsector).

Se traslada el aporte a las empresas del Área Metropolitana de Bucaramanga, generando un espacio de conocimiento en los procesos de personal, tales como los de planeación y selección de personal, análisis de cargos, compensaciones y planes de crecimiento o desarrollo de personal en temas como capacitación, plan de carreras y gerencia del desempeño.

Algunos de los temas sugeridos para futuras investigaciones es la realización de este tipo de estudios en otros niveles jerárquicos dentro de la organización o en un subsector; además, podrían hacerse ejercicios similares en la comunidad educativa para analizar la producción intelectual o la creación de empresas spin off de acuerdo a los perfiles de los investigadores. Igualmente, sería valioso que los resultados fueran analizados desde otras disciplinas como la psicología organizacional y desde la interdisciplinariedad entre grupos de investigación o la investigación puntual de los perfiles en la productividad, la producción intelectual con los perfiles de los investigadores, la creación de empresas spin off y el perfil de los investigadores, etc.

\section{Referencias}

Bass, B. (1985). Leadership and performance beyond expectations, New York: Free Press.

Bass, B. (1990). Handbook of leadership: A Survey of Theory and Research. New York: Free Press.

Bass, B. (1997). Does the transactional-transformational leadership paradigm transcend organizational and national boundaries? American Psychologist, 52 (2), 130-1391.

Bass, B., Avolio, B., Jung, D., Berson, Y. (2003). Predicting unit performance by assessing transformational and transactional leadership. Journal of applied psychology, 88(2), 207-218. 
Becker, G. (1964). Human Capital, (1 ${ }^{\mathrm{a}}$ ed.) NBER. Nueva York, Estados Unidos: Columbia University Press.

Becker, G. (1983). El capital humano. (2a ed.) Madrid, España: Alianza Editorial S.A.

Burns, J. (1978). Leadership. New York: Harper y Row.

Carpenter, M. y Geletkanycz, M. y Sanders, G. (2004). Upper echelons research revisited: antecedents, elements, and consequences of top management team Composition. Journal of Management, 30(6) 749-778.

Cartwright, D. y Zander, A. (1960). Group Dynamics: research and theory. Nueva York: Harper and Row.

Castro, A. y Nader, M. (2004). Valoración de un programa académico y militar de un programa de cadetes argentino, Estudio de valores y de liderazgo. Revista Iberoamericana de Evaluación y Diagnóstico Psicológico, 10(17), 29-45.

Castro, A., y Casullo, M. (2005). Personality styles, coping strategies and intelligence as predictors of military cadets' academic careers. Anuario de Psicología, 36 (2), $197-210$.

Evans, M. (1970). The effects of the supervisory behavior on the path goal relationship. Organizational Behavior and Human Performance, 5, 277-298.

Eyupoglu, S y Saner, T. (2011). Who is she? The turkish cypriot female entrepreneur. African journal of business management, 5(15), 6247.6255

Fiedler, F. (1967). A theory of leadership effectiveness. Nueva York: McGraw-Hill.

Gundry, L., y Welsch, H. (2001). The ambitions entrepreneur: High growth strategies of women-owned enterprises. Journal of Business Venturing, 16 (5), 453-470.

Hambrick, D. y Mason, P. (1984). Upper echelons: the organization as a reflection of its top managers. The Academy of Management Review, 9(2), 193-206.

Hemphill, J., y Coons, A. (1957). Development of the leader behavior description questionnaire. En R Hemphill, J., Coons, A. (1957). Development of the leader behavior description questionnaire. En R. Stogdill y A. Coons (eds.), Leader Behavior: Its Description and Measurement. Columbus $(\mathrm{OH})$ : Bureau of Business Research of Ohio State University.

Hersey, P., Blanchard, K. y Johnson, D. (1969). Management of Organizational. Englewood Cliffs: Prentice Hall.

House, R. (1971). A path goal theory of the leader effectiveness. Administrative Science Quarterly, 16, 312-328. 
House, R. (1977). A 1976 theory of charismatic leadership, en J. Hunt y L. Larson (Eds.).

House, R., y Dessler, G. (1974a). The path goal theory of leadership: Some post hoc and priori test. En J. Hunt y L. Larson (Eds.) Contingency Approaches in Leadership. Carbondale: Southern Illinois University Press.

House, R., y Mitchell, T. (1974b). Path-goal theory of Leadership. Contemporary Business, 3, 81-98.

Kaminskas, G., Bartkus, E., Pilinkus, D. (2011). Leadership as a reciprocity of leader and followers. Inzinerine Ekonomika - Engineering Economics, 22(2), 175-185.

Kirkpatrick, S, y Locke, E. (1991). Leadership: Do traits matther? The Executive, 5, 48-60.

Lewis, W. A. (1976). Teoría del Desarrollo Económico. Bogotá, Colombia: FCE.

Lord, R., y De Vader, C., y Alliger, G. (1986). A meta-analysis of the relation between personality traits and leadership perceptions: an application of validity generalization procedures. Journal of Applied Psychology, 71, 402-410.

Mann, R. (1959). Personal factors associated with leadership: a survey of the literature, Journal of Psychology, 25, 241-270.

Masten, A., y Coastworth, D. (1998). The development of competence in favorable an unfavorable environments. American Psychologist, 53, 205-220.

Mincer. (1974). Schooling Experience and Earnings. National Bureau Economics Research. New York, Estados Unidos: Columbia University Press.

Molero, F., y Cuadrado, I., Navas, M. y Morales, J. (2007). Relations and effects of transformational leadership: A comparative analysis with traditional leadership styles. Spanish journal of psychology, 10 (2). 358-368.

Moriano, J., y Molero, F., y Mangin, L. (2011). Liderazgo auténtico. Concepto y validación del cuestionario ALQ en España. Psicothema, 23 (2), 336-341.

Nader, M. y Castro Solano, A. (2010). Teorías implícitas de liderazgo, LMX y bienestar laboral: generalización de un modelo teórico. Revista de Psicología de la Pontificia Universidad Católica de Perú, 28 (2), 228-258.

Nadkami, S. y Hermann, P. (2010). Ceo personality, strategic flexibility, and firm performance: the case of the Indian business process outsourcing industry. Academy of Management Journal, 53 (5), 1050-1073.

Northouse, P. (2004). Leadership: Theory and Practice. Thousand Oaks, CA. Sage.

Ogliastri, E. (2005). Liderazgo Organizacional en Colombia: Un Estudio Cualitativo. Revista Eafit, 15, 1-18. 
Pedraja, L., y Rodríguez, E., y Rodríguez, J. (2006). Leadership Styles and Effectiveness: A study of a small firms in Chile. Interciencia, 31 (007), 500-504.

Rauch, A. y Frese, M. (2007). Let's put the person back into entrepreneurship research: a meta-analysis on the relationship between business owners' personality traits, business creation, and success. Journal of Work and Organizational Psychology, 16 (4), 353-385.

Schultz, W. (1959). Investment in man: an economist's view. Soc. Serv. Rev, 33, 109117.

Smith, A. (1983). Investigación de la naturaleza y causas de la riqueza de las naciones. Editorial Orbis S.A. Barcelona. Traducido de la Obra Original Publicada en Londres 1975, Inglaterra.

Spanos, Y, y Lioukas, S. (2001). An examination into the causal logic of rent generation: contrasting porter's competitive strategy framework and the resourcebased perspectiv. Strategic Management Journal, 22(10), 907-934.

Stogdill, R. (1948). Personal factors associated with leadership: a survey of the literature. Journal of Psychology, 25, 35-71.

Stogdill, R. (1963). Manual for the LBDQ-Form XII. Columbus (OH): Bureau of Business Research of Ohio State University.

Wang, h., Tsui, A., Xin, K. (2011). Leadership behaviors, organizational performance, and employees' attitudes. The Leadership Quarterly, 22, 92-105.

Yang, L., Wu, K., Wang, F., Chin, P. (2012). Relationship among project manager's leadership style, team interaction and project performance in Taiwanesse server industry. Quality \& Quantity, 46(1), 207-219. 